\title{
BOUNDED INDEX, ENTIRE SOLUTIONS OF ORDINARY DIFFERENTIAL EQUATIONS AND SUMMABILITY METHODS
}

\author{
G.H. FRICKE \\ Department of Mathematics \\ Wright State University \\ Dayton, Ohio 45431 \\ RANJAN ROY \\ Department of Mathematics \\ State University of New York \\ College at Plattsburgh \\ Plattsburgh, New York 12901 \\ and \\ S.M. SHAH \\ Department of Mathematics \\ University of Kentucky \\ Lexington, Kentucky 40502 \\ (Received December 17, 1980)
}

ABSTRACT. A brief survey of recent results on functions of bounded index and bounded index summability methods is given. Theorems on entire solutions of ordinary differential equations with polynomial coefficients are included.

KEY (1)ORUS AND PHPASES. Bounded Index, Summability, Vikferential Equations, Entere Solutions.

1980 Mathematics Subject Classification Codes. 30015, 40005. 34A20, 34 A40.

1. INTRODUCTION AND DEFINITIONS.

DEFINITION 1.1. An entire function $\mathrm{f}: \phi \rightarrow \phi$ is said to be of bounded index (b.i.) if there exists an integer $N \geq 0$ such that

$$
\max _{0 \leq j \leq I I} \frac{\left|f^{(j)}(z)\right|}{j !} \geq \frac{\left|f^{(n)}(z)\right|}{n !}
$$

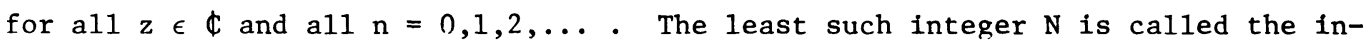
dex of $f$ (see Lepson [30], Shah [40]). 
DEFINITION 1.2. An entire function $f$ is said to be of bounded value distribution (b.v.d.) if for every $r>0$, there exists a fixed integer $P(r)>0$ such that the equation $f(z)=w$ has never more that $P(r)$ roots in any disc of radius $r$ and for any $w \in \notin$ (see Hayman $[16,17])$.

A survey of the properties of functions of b.i., and of b.v.d., and a list of references published up to 1975 and some up to 1076, are given in [40].

In Section 2 we give some extensions of these concepts to meromorphic functions [3]. If an entire function is of b.i. N, then its growth is $(1 ; \mathrm{N}+1)([17]$, [37], [13]). In Section 3 we study extensions of (1.1) suitable for entire functions of finite order. Here we show, following Hennekemper [19], that if $\mathrm{E}[\rho, \infty)$ be the set of all entire functions of order not exceeding $\rho$, and not of maximal type order $\rho$, and if $f$ be of $b . i . N$, then $\left(f, f^{1}, \ldots, f^{(N)}\right)_{1}=E[1, \infty)$, where the left side denotes the ideal in $E[1, \infty)$ finitely generated by $f, f^{1}, \ldots, f(N)$. In Section 4 we consider entire solutions of linear differential equations with polvnomial coefficients and give theorems relating to the property of b.i., and bounds on the index $\mathrm{N}_{f}$ of an entire solution $f$ of bounded index, and also a bound on the growth rate of $f$. Here and in the preceding section we have included some new results and shorter proofs of some known results. (Theorems and Examples without accompanying references are new.) The summability methods related to bounded index property are given in Section 5. Finally in Section 6 we give some recent results, on functions defined by nirichlet series and on functions of several variables.

2. FUNCTIONS OF B.I. AND B.V.D.

It is known that if $f$ is of b.i., then it is of exponential type ([17], [13]) but functions of exponential type need not be of $b . i$. In fact there exist functions of exponential type and having simple zeros and of unbounded index [39]. The following theorem gives a necessary and sufficient condition for an entire function of exponential type to be of b.i.

THEOREM 2.1. (Fricke [10]). Let $f$ be an entire function of exDonential type. Then $f$ is of $b . i$. if and only if for each $d>0$, there exists $M=M(d)>0$ such that $\left|f^{\prime}(z)\right| \leq M|f(z)|$ for all $z$ with $\left|z-a_{n}\right| \geq d$ for all $n$. Here $a_{n}^{\prime}$ 's denote 
the zeros of $f$.

The bound $M$ depends on the closeness to the zeros of $f$. If we now examine the behavior of the logarithmic derivative near a zero $a_{t}$ of order $m$, we see that

$$
\frac{f^{\prime}(z)}{f(z)}-\frac{m}{z-a_{t}}
$$

is bounded in a neighborhood of $a_{t}$; that is

$$
\left|\frac{f^{\prime}(z)}{f(z)}\right| \leq \frac{m+1}{\left|z-a_{t}\right|}
$$

for $z$ sufficiently close to $a_{t}$. This simple observation is used to improve Theorem 2.1. For an entire function $f$, let

$$
R(z)=R_{f}(z)=\max \left[\{1\} \cup\left\{\frac{1}{\left|z-a_{n}\right|} \mid n=1,2, \ldots\right\}\right]
$$

where the $a_{n}$ 's are the zeros of $f$.

THEOREM 2.2. (Fricke [10]). An entire function of finite order is of b.i. If and only if there exists a constant $M>0$ such that

$$
\left|f^{\prime}(z)\right| \leq \operatorname{MR}(z)|f(z)| \quad \text { for } a 11 z \text {. }
$$

The proof of Theorem 2.2 makes use of the following lemma [10]: If f is an entire function of finite order such that for some $M>0$,

$$
\left|f^{\prime}(z)\right| \leq \operatorname{MR}(z)|f(z)| \quad \text { for al1 } z \text {, }
$$

then there exists an integer $N$ such that any closed disk of radius 1 contains at most $N$ zeros of $f$.

Beauchamp [3] extended tha basic ideas in Theorem 2.2 to meromorphic functions. To present his results we need the following notations.

DEFINITION 2.3. Let $A \subset \mathbb{} \cup\{\infty\}$. A function $f$ meromorphic on $\downarrow$ is said to be $A-b . v . d$. If there exists an integer $P$ such that for any $w \in A, f(z)-w$ has at most $P$ zeros in any disk of radius 1 . If $w=\infty \in A$ this implies that $f$ has at most $P$ poles in any disk of radius 1 . If $A=\mathbb{C} U\{\infty\}$, we simply say that $f$ is b.v.d.

DEFINITION 2.4. A function $f$ meromorphic on $C$ is said to be D.I. (differential inequality) if

(i) $f$ is $\{\infty\}-b \cdot v \cdot d$. 
(ii) f satisfies an inequality of the form

$$
\left|f^{(N+1)}(z)\right| \leq R(z) \max _{0 \leq j \leq N}\left|f^{(j)}(z)\right|
$$

for all $\mathrm{z} \in \phi \backslash P$, where $\mathrm{N}$ is a positive integer,

$$
P=\left\{b_{n} \in C \mid f \text { has a pole at } b_{n}\right\},
$$

and $R(z)$ is a real valued function with $R(z) \geq 1$ and $R(z)$ decreasing

with respect to the distance of $z$ to the poles, that is,

$$
R(z)=D[d(z, P)] \text { where } D:(0, \infty] \rightarrow[1, \infty) \text { is a decreasing }
$$

function. (If $\mathrm{f}$ is entire we may consider $\mathrm{R}(\mathrm{z})$ to be constant.)

For entire functions and for $R(z) \equiv C$, Hayman [17] showed that the above condition is equivalent to bounded index.

DEFINITION 2.5. A function $f$ meromorphic on $\phi$ is said to be L.D.I. (1ogarithmic differential inequality) if

(i) $f$ is $\{0, \infty\}-b \cdot v \cdot d$.

(ii) the logarithmic derivative satisfies

$$
\left|\frac{f^{\prime}(z)}{f(z)}\right| \leq L(z) \quad \text { for all } z \in \phi \backslash D
$$

where $D$ is the set of zeros and poles of $f$ and $L(z)$ is a decreasing function with respect to the distance of $z$ to $D$ and $L(z) \geq 1$.

Using the above definitions, Beauchamp was able to obtain among other results the following:

THEOREM 2.6. (Beauchamp [3]). A function $\mathrm{f}$ meromorphic on $\phi$ is D.I. if and only if it is L.D.I.; in fact if $\mathrm{f}$ is D.I. then $\mathrm{R}(z)$ in Definition 2.4 may be chosen to be of the form $R(z)=M\left[\max \left\{1, \frac{1}{d(z, P)}\right\}\right]^{K}$, where $M$ is a constant $\geq 1$, and $\mathrm{N}$ and $\mathrm{K}$ are integers with $1 \leq \mathrm{K} \leq \mathrm{N}$.

THEOREM 2.7. (Beauchamp [3]). A function $f$ meromorphic on $\phi$ is b.v.d. if and only if $f^{\prime}(z)$ is D.I.

THEOREM 2.8. (Beauchamp [3]). Let $\mathrm{f}$ and $\mathrm{g}$ be D.I. Then

(i) the function $\frac{1}{\mathrm{f}}$ is D.I.

(ii) The product $h=f g$ is $D . I$. 
THEOREM 2.9. (Beauchamp [3]). Let $f$ be D.I. Then $f$ is of order not exceeding 2 and finite type.

In [3] Beauchamp also examines and obtains similar results for functions meromorphic on the unit disk. Here $R(z)$ and $L(z)$ depend not only on the distance to the zeros, respectively zeros and poles, but also on the distance to the boundary of the unit disk.

\section{BOUNDED INDEX CONCEPT FOR FUNCTIONS OF FINITE ORDER.}

It is known that if $\mathrm{f}$, entire on $\phi$, is of order $>1$ or of order 1 and maximal type then the growth rate of the derivative may be larger than that of the function (Shah [36], Vijayaraghavan [46], Kövari [28]) and so inequalities of the type (1.1) may not hold. To overcome this difficulty both sides of the inequality (1.1) are multiplied by a factor. Thus we have:

DEFINITION 3.1. (Beauchamp [3]). Let $f$ be entire on $\phi$ and $\gamma \geq 0$. The function $\mathrm{f}$ is $\gamma$-b.i. ( $\gamma$-bounded index) if there exist a number $r_{0}>0$ and an integer $\mathrm{N} \geq 0$ such that

$$
\frac{|f(n)(z)|}{n !|z|^{n \gamma}} \leq \max _{0 \leq \nu \leq N} \frac{|f(\nu)(z)|}{\nu !|z|^{\nu \gamma}}
$$

for all $|\mathrm{z}| \geq \mathrm{r}_{\mathrm{o}}$ and $\mathrm{n} \geq \mathrm{N}$.

This definition is an extension of (1.1) to entire functions of finite order. If $f$ is of $b . i . N$ then $f$ is of growth $(1, N+1)([40])$. Here we have THEOREM 3.2. (Beauchamp [3]). If $f$ is $\gamma-b . i$. satisfying (3.1) then $f$ is of growth $\left(\gamma+1, \frac{\mathrm{N}+1}{\gamma+1}\right)$

Another extension of (1.1) is as follows:

DEFINITION 3.3. (Hennekemper [19]). An entire function $f$ is said to be of bounded $\alpha$-index $\mathrm{N}$ if $\alpha \in \mathrm{R}$ and $\mathrm{N}$ is the smallest integer such that for all $\mathrm{n}$,

$$
\max _{0 \leq j \leq N}\left\{\frac{|f(j)(z)|}{j !}\right\} \geq \frac{|f(n)(z)|}{n !} \text { for all z: }|z|<1,
$$

and

$$
\max _{0 \leq j \leq N}\left\{\frac{|f(j)(z)|}{j !}\right\}|z|^{\alpha j} \geq \frac{\left|f^{(n)}(z)\right|}{n !}|z|^{\alpha n} \text { for all } z:|z| \geq 1
$$


This definition is a slight variation of the one given by G. Frank [6], and is used by Hennekemper to prove Theorem 3.4 below.

Let $E[\rho, \infty)$ be the set of all entire functions which are of order not exceeding $\rho(0<\rho<\infty)$ and not of maximal type order $\rho$, that is,

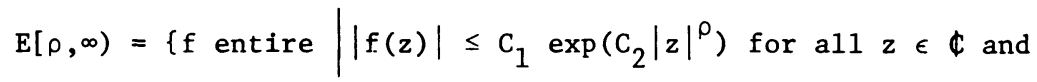

$$
\begin{aligned}
& \text { constants } \left.C_{1}=C_{1}(f) \text { and } C_{2}=C_{2}(f)\right\} \text {. }
\end{aligned}
$$

Let $f_{1}, f_{2}, \ldots, f_{n} \in E[\rho, \infty)$ and denote by $\left(f_{1}, f_{2}, \ldots, f_{n}\right)_{\rho}$ the ideal in $E[\rho, \infty)$

finitely generated by $f_{1}, f_{2}, \ldots, f_{n}$.

THEOREM 3.4. (Hennekemper [19]). Let $\mathrm{f} \in \mathrm{E}[\rho, \infty)$ be of bounded $\alpha$-index $\mathrm{N}$ and let $\varepsilon>0$. Then

$$
\left(\mathrm{f}, \mathrm{f}^{\prime}, \ldots, \mathrm{f}^{(\mathrm{N})}\right)_{\rho+\varepsilon}=\mathrm{E}[\rho+\varepsilon, \infty) .
$$

We give below a different proof of this theorem when $\rho=1$ and $\alpha=0$. For another proof see [19].

THEOREM 3.5. Let $\mathrm{f}$ be an entire function of $\mathrm{b} .1 . \mathrm{N}$, not identically zero.

Then

$$
\left(\mathrm{f}, \mathrm{f}^{\prime}, \ldots, \mathrm{f}^{(\mathrm{N})}\right)_{1}=\mathrm{E}(1, \infty) \text {. }
$$

PROOF. Since $f$ is of $b . i . N$, we have for any $j$

$$
\left|f^{(j)}(z)\right| \leq j ! \max _{0 \leq k \leq N}\left\{\left|f^{(k)}(z)\right|\right\} \quad \text { for all } z \in \mathbb{Q} \text {. }
$$

Thus, for $\mathrm{C}=(\mathrm{N}+1)$ !

$$
\left|f^{(j)}(z)\right| \leq C \sum_{k=0}^{N}\left|f^{(k)}(z)\right|
$$

for al1 $\mathrm{z}$, and $\mathrm{j}=1,2, \ldots, \mathrm{N}+1$. Let $\alpha \in \Phi,|\alpha|=1$ and

$$
G(r)=\sum_{k=0}^{N}\left|f^{(k)}(\alpha r)\right| \text {. }
$$

Then $G(r)$ is continuous and piecewise continuously differentiable. Also because of the definition of b.i.,

$$
\max _{0 \leq \ell \leq N}\left|f^{(l)}(z)\right|>0 \text { and thus } G(r)>0 \text { for } r \geq 0 .
$$

Hence for all $\mathrm{r}$, except possibly for a set of measure zero, 


$$
\begin{aligned}
\left|G^{\prime}(r)\right| & =\left|\sum_{k=0}^{N} \frac{d f^{(k)}(\alpha r) \mid}{d r}\right| \leq \sum_{k=0}^{N}\left|f^{(k+1)}(\alpha r)\right| \\
& \leq G(r)+\left|f^{(N+1)}(\alpha r)\right| \\
& \leq G(r)+C G(r) .
\end{aligned}
$$

Thus

$$
\left|G^{\prime}(r)\right| / G(r) \leq c+1, \quad G^{\prime}(r) / G(r) \geq-(c+1) .
$$

Hence

$$
\log \frac{G(r)}{G(0)}=\int_{0}^{r} \frac{G^{\prime}(x)}{G(x)} d x \geq-(C+1) r .
$$

Now if we let $C_{1}=1 / G(0)$ and $C_{2}=C+1$, then for all $r \geq 0$

$$
\frac{G(r)}{G(0)}=C_{1} G(r) \geq \exp \left(-C_{2} r\right)
$$

and thus for all $\mathrm{r} \geq 0$

$$
C_{1} \exp \left(C_{2} r\right) G(r) \geq 1 \text {. }
$$

Since arg $\alpha$ was arbitrary, we obtain by considering $z=\alpha r$,

$$
1 \leq C_{1} \exp \left(C_{2}|z|\right) \underset{j=0}{N}\left|f^{(j)} \quad(z)\right|
$$

for all z. The proof can now be concluded by applying the following ([21], [23], [19])

LEMMA. Let $f_{o}, f_{1}, \ldots, f_{n} \in E[\rho, \infty)$. The $\left(f_{o}, f_{1}, \ldots, f_{n}\right)_{\rho}=E[\rho, \infty)$ if and only if there exist $\mathrm{C}_{1}, \mathrm{C}_{2}>0$ such that

$$
1 \leq C_{1} \exp \left(C_{2}|z|^{\rho}\right)\left\{\sum_{j=0}^{n}\left|f_{j}(z)\right|\right\}
$$

for all $z \in \mathbb{\$}$.

\section{ENTIRE SOLUTIONS OF DIFFERENTIAL EQUATIONS.}

(i) Consider the differential equation (d.e.)

$$
L_{n}(w, a)=a_{0} w^{(n)}+a_{1} w^{(n-1)}+\ldots+a_{n} w=0, a_{0} \neq 0
$$

and the d.e.

$$
L_{n}(w, P)=P_{0}(z) w^{(n)}+P_{1}(z) w^{(n-1)}+\ldots+P_{n}(z) w=0
$$

where $a_{k} \in \mathbb{Q}, P_{k}$ are all polynomials and 


$$
P_{k}(z)=A_{k} z^{\alpha}(1+o(1)),|z| \rightarrow \infty .
$$

The following results are known.

THEOREM 4.1. (Shah [37]). All solutions of the equation $L_{n}(w, a)=0, a_{k} \in \mathbb{d}$, are entire functions of b.i. and b.v.d.

There it is shown that every solution $f(z)$ is of $b .1$, and a bound on the index of $\mathrm{f}$ is also given. By differentiating (4.1), one sees easily that every solution is of b.v.d.

THEOREM 4.2. (Shah [38]). If

$$
\operatorname{deg} P_{0} \geq \max _{1 \leq k \leq n} \operatorname{deg} P_{k},
$$

then all those solutions of the equation $L_{n}(w, P)=0$, which are entire functions, are of b.1. and b.v.d.

Extensions of this theorem are given by Fricke and Shah ([11],[13]). Bounds for the index $N_{f} \equiv N(f)$ of an entire solution $f$ of $(4.2)$ are known in some particular cases ([31], [22], [44]). Note that Theorem 4.2 implies that any entire solution $w$ is of exponential type $N\left(f_{w}\right)+1$. A bounded index on the growth rate of a solution, without the hypothesis $(4.4)$, is given in the next theorem. Write

$$
\begin{gathered}
\gamma=\max _{1 \leq k \leq n} \frac{\alpha_{k}-\alpha_{0}}{k}, \alpha_{k}=-\infty \text { if } P_{k} \equiv 0, \\
\ell_{k}= \begin{cases}A_{k} & \text { if } \alpha_{k}-\alpha_{0}=k \gamma \\
0 & \text { if } \alpha_{k}-\alpha_{0}<k \gamma .\end{cases}
\end{gathered}
$$

THEOREM 4.3. (Beauchamp [3]). If $\mathrm{f}$ is an entire solution of the equation $L_{n}(w, P)=0$, and if $\gamma \geq 0$, then $f$ has growth

$$
\left\{\gamma+1,\left(\sum_{k=1}^{n}\left|\ell_{k}\right|\right) /(\gamma+1)\left|A_{0}\right|\right\}
$$

The following examples show that the growth bound (4.5) cannot, in general, be improved.

EXAMPLE 4.4. (Beauchamp [3]). Let $f(z)=\exp \left(z^{k}\right)$ where $k \geq 1$ is an integer. Then $f$ satisfies the equation

$$
w^{\prime}-k z^{(k-1)} w=0
$$


Here $\gamma=k-1, A_{0}=1, \ell=-k$ and $f$ has growth $(k, 1)$.

EXAMPLE 4.5. The Bessel function $J_{n}(z)$ of order $n$, where $n$ is a positive or negative integer or zero, satisfies the equation

$$
z^{2} w^{\prime \prime}+z w^{\prime}+\left(z^{2}-n^{2}\right) w=0 \text {. }
$$

Here $\gamma=0, A_{0}=1, \ell_{1}=0, \ell_{2}=1$ and $J_{n}$ has growth $(1,1)$.

(ii) We now consider one type of converse of Theorem 4.2. We seek a set of entire functions $g_{k}$ such that every entire function $f$ of $b . i$. satisfies a linear d.e. of the form

$$
f^{(n)}+g_{n-1} f^{(n-1)}+\ldots+g_{o} f=0
$$

where $g_{0}, g_{1}, \ldots, g_{n-1}$ are entire functions.

THEOREM 4.6. (Hennekemper [19],[20]). Let $f$ be of b.i. N. Then $f$ satisfies a linear differential equation of the form

$$
f^{(N+1)}+g_{N} f^{(N)}+\ldots+g_{o} f=0
$$

with $\mathrm{g}_{\mathrm{k}} \in \mathrm{E}[1, \infty)$.

COROLLARY 4.7. (Hennekemper [20]). Any entire function of exponential type can be written as the difference of two functions each satisfying a linear d.e. of order $\mathrm{N}$ with coefficients from $\mathrm{E}[1, \infty)$.

For the proof of Theorem 4.6 we on 1 y need to note that (Theorem 3.5)

$$
f^{(N+1)} \in E[1, \infty)=\left(f, f^{1}, \ldots, f^{(N)}\right)_{1} \text {. }
$$

The Corollary relies on the fact that any function of exponential type can be written as the sum of two functions of b.i. ([42]).

REMARK. Simple examples such as $\operatorname{Sin} z$, Cos $z(N=1), e^{z}(N=0)$ show that the order of the equation $(4.6)$ is best possible.

(iii) Another type of converse to Theorem 4.2 is as follows:

THEOREM 4.8. If all $n$ solutions of d.e. $L_{n}(w, P)=0$ are entire functions of exponential type, then deg $\mathrm{P}_{0} \geq \operatorname{deg} \mathrm{P}_{k}$, for $\mathrm{k}=1,2, \ldots, \mathrm{n}$; and thus the solutions are of b.i. and b.v.d.

We sha11 deduce this from

THEOREM 4.9. If all $\mathrm{n}$ solutions are entire functions and

$$
\operatorname{deg} \mathrm{P}_{0}<\max _{\mathrm{k}>0} \operatorname{deg} \mathrm{P}_{k}
$$


then at least one solution $w$ is of order $\lambda_{1}$ where

$$
\lambda_{1}=1+\max _{k>0} \frac{\alpha_{k}-\alpha_{0}}{k}>1 .
$$

PROOF. This result follows easily from the results and methods of Knab [24-27], Wittich [47], Posch1 [34], Boehmer [4] and Frank [5]. We sketch briefly the main argument. By our hypothesis

$$
\alpha_{0}<g \equiv \max _{i=1}^{n}\left\{\alpha_{i}+i\right\} .
$$

Hence there is a region $S$, the plane cut along a half ray, in which a single-valued branch $W$ of the solution $w$ can be defined with the property that if

$$
M(r, W)=\max _{|z|=r}\{|W(z)|, z \in S
$$

then

$$
\lambda(W)=\lim _{r \rightarrow \infty} \sup \frac{\log \log M(r, W)}{\log r}>0 .
$$

Since all the solutions, by our hypothesis, are entire, we can choose the branch to be the solution itself and this implies that there is at least one transcendental solution with positive order (see also Ince [22, 424-427]).

Now we use Wiman-Valiron central index method (cf: Wittich [47, 4-11; 65-73], Valiron $[45,105-109 ; 177-181])$. For the transcendental solutions

$$
\frac{\mathrm{w}^{(\mathrm{k})}(\zeta)}{\mathrm{w}(\zeta)} \sim\left(\frac{\mathrm{N}}{\zeta}\right)^{\mathrm{k}}, \mathrm{k}=1,2, \ldots ; \text { as } \zeta \rightarrow \infty \text {, }
$$

except for a set of values of $r=|\zeta|$ of finite logarithmic measure. Here $N=N(r)$ is the central index and the points $\zeta$, on $|z|=r$, are the points at which the maximum modulus is attained:

$$
w(\zeta)=\max _{|z|=r}|w(z)| .
$$

We substitute this asymptotic relation in (4.2) and put $N=1 / Y, \zeta=1 / \mathrm{X}$ and then the equation (4.2) becomes

$$
\sum_{k=0}^{n} A_{k} X^{m_{k}} Y^{k}\left(1+\eta_{k}(X)\right)=0
$$

where $m_{k}=k+n+\alpha_{0}-\alpha_{k}-k, k=\max _{k>0}\left(\alpha_{k}-\alpha_{0}\right)$ and $\eta_{k} \rightarrow 0$ as $x \rightarrow 0$. One next constructs a Newton's polygon (cf: [33], [47; 67-72]) with points $\left(k, m_{k}\right.$ ) and it follows (cf: Knob $[25,27]$ ) that the negative slopes of the sides of the polygon 
give the orders of the solutions and that the negative of the slope of the side through $\left(0, m_{0}\right)$ is the order of the solution of maximal growth. The negative of this slope is in fact $1+\max _{k>0} \frac{\alpha_{k}-\alpha_{0}}{k}>1$.

REMARKS. (i) Note that the condition (4.7) implies (4.9). We require (4.7) for the concluding part of the proof of Theorem (4.9).

(1i) The following example shows that the hypothesis, in Theorem 4.9, that all solutions are entire is necessary.

EXAMPLE 4.10. [40].

$$
z w^{\prime \prime}+\left(z^{2}-z-\frac{1}{2}\right) w^{\prime}-\left(z^{2}-\frac{1}{2}\right) w=0 \text {. }
$$

Here (4.7) is satisfied. One solution $w_{1}(z)=e^{z}$ is entire but the second solution is not entire, and the conclusion of Theorem (4.9) does not hold.

We now give two more examples.

EXAMPLE 4.11. [5, p. 61-62]).

$$
\begin{aligned}
\left(2 z^{2}-2 z-1\right) w^{\prime \prime} & +\left(-8 z^{3}+6 z^{2}+2 z+3\right) w^{\prime \prime}+\left(8 z^{4}-10 z^{2}+2 z+7\right) w^{\prime} \\
+ & \left(-8 z^{4}+8 z^{3}+2 z^{2}-2 z-9\right) w=0 .
\end{aligned}
$$

Here all three solutions are entire functions:

$$
w_{1}=e^{z^{2}-z}, w_{2}=e^{z^{2}+z}, w_{3}=e^{z}
$$

Here

$$
\begin{gathered}
\alpha_{0}=2, \alpha_{1}=3, \alpha_{2}=4, \alpha_{3}=4, m_{0}=5, m_{1}=3, m_{2}=1, m_{3}=0 \\
\lambda_{1}=1+\max _{k>0}\left\{\frac{\alpha_{k}-\alpha_{0}}{k}\right\}=2 .
\end{gathered}
$$

EXAMPLE 4.12

$$
w^{\prime \prime}-2 z w^{\prime}+2 n w=0
$$

Here both solutions are entire functions, one a polynomial (Hermite polynomial, when normalized) and the second, a transcendental function of order

$$
\lambda_{1}=1+\max \{1,0\}=2 \text {. }
$$

Here $\mathrm{m}_{\mathrm{o}}=3, \mathrm{~m}_{1}=1, \mathrm{~m}_{2}=1$.

(iii) Frank and Frank and Mues ([6-8]; see also [40]) introduce a function I(r,f) to define a function of b.i. Consider the Taylor expansion of an entire function 
f about a point a:

$$
f(z)=\sum_{n=0}^{\infty} \frac{f^{(n)}(a)}{n !}(z-a)^{n}, a \in \phi ;
$$

and let $k_{a}$ be the largest nonnegative integer such that

$$
\frac{\left|f_{a}^{\left(k_{a}\right)}(a)\right|}{\left(k_{a}\right) !} \geq \frac{\mid f_{(a)}^{(n)}}{n !} \text { for } n=0,1,2, \ldots \text {. }
$$

Define $I(r, f)=\sup _{\mid a} \mid \leq r k_{a}$.

If $\lim \sup _{r \rightarrow \infty} I(r, f)<\infty$ then $f$ is said to be of $b . i$. This definition is equivalent to (1.1). Frank and Mues [8] showed that if $\mathrm{f}$ is an entire function of finite order $\rho$, then

$$
\max (0, \rho-1) \leq \limsup _{r \rightarrow \infty} \frac{\log ^{+} I(r, f)}{\log r} \leq \rho .
$$

We now state an extension of this theorem.

THEOREM 4.13. (a) Suppose the hypothesis of Theorem 4.9 is satisfied. Then there is a solution w of order $\lambda_{1}$ given by (4.8). The index $I(r, w)$ of this solution w satisfies

$$
\lim _{\mathrm{r} \rightarrow \infty} \frac{\log ^{+} \mathrm{I}(\mathrm{r}, \mathrm{w})}{\log \mathrm{r}}=\lambda_{1}-1 \text {. }
$$

(b) If $\operatorname{deg} \mathrm{P}_{\mathrm{o}} \geq \max _{\mathrm{k} \geq 0} \operatorname{deg} \mathrm{P}_{k}$, then

$$
\lambda_{1}=1+\max _{\mathrm{k}>0} \frac{\alpha_{k}-\alpha_{0}}{k} \leq 1
$$

and any entire solution of (4.2) satisfies

$$
\lim _{r \rightarrow \infty} I(r, w)<\infty .
$$

We omit the proof of (a) which is similar to the proof of Theorem 2 of [7]. The second part (b) is a restatement of Theorem 4.8 .

(iv) Heath considers vector-valued entire functions of b.i. and proves a result, similar to Theorem 4.2 , for vector equations.

THEOREM 4.14. (Heath [18]). If $F$ is an entire solution of $F^{\prime}=A F+Q$ where $A=\left[r_{i j}\right]$ is a matrix whose entries are rational functions which are bounded at infinity and $Q$ is a vector whose entries are rational functions which are bounded at infinity, then $F$ is a function of bounded index. 


\section{BOUNDED INDEX AND SUMMABILITY METHODS.}

We begin with definitions and notations. A sequence $X=\left\{x_{k}\right\}_{0}^{\infty}$ of complex numbers is an entire sequence if $\Sigma_{k=0}^{\infty}\left|x_{k}\right| q^{k}$ converges for every positive integer $q$, that is, if $f(z)=\Sigma_{k=0}^{\infty} x_{k} z^{k}$ is an entire function. We will denote the set of entire sequences by $E$. An entire sequence $x=\left\{x_{k}\right\}_{0}^{\infty}$ is of bounded index if $f(z)=$ $\Sigma_{k=0}^{\infty} X_{k} z^{k}$ is of b.i., and we denote the set of sequences of b.i. by $B$.

Furthermore, let $c_{0}$ be the set of null sequences, $c$ the set of convergent sequences, $\ell$ be the set of absolutely convergent sequences, that is

$$
l=\left\{X=\left\{X_{k}\right\}_{0}^{\infty}: \sum_{k=0}^{\infty}\left|x_{k}\right|<\infty\right\}
$$

and let

$$
E *=\left\{X=\left\{X_{k}\right\}_{0}^{\infty}:\left|x_{k}\right|^{\frac{1}{k}} \text { is bounded }\right\}
$$

and

$$
x=\left\{X=\left\{x_{k}\right\}_{0}^{\infty}:\left(k !\left|x_{k}\right|\right)^{\frac{1}{k}} \rightarrow 0 \text { as } k \rightarrow \infty\right\} .
$$

Then $x$ can be regarded as the collection of functions $f(z)=\Sigma_{k=0}^{\infty} x_{k} z^{k}$ of exponential type of order 1 and type 0 .

If $R$ and $S$ are collections of sequences, then a matrix $A=\left(a_{n, k}\right)$ is an $R-S$ method if it maps sequences of $R$ to sequences in $S$.

The Taylor matrix $T(r)=\left(a_{n, k}\right)$ is defined by $[35, p .60]$

$$
a_{n, k}=\left\{\begin{array}{cl}
\left(\begin{array}{l}
k \\
n
\end{array}\right)(1-r)^{n+1} r^{k-n} & \text { for } k \geq n \\
0 & \text { otherwise } .
\end{array}\right.
$$

THEOREM 5.1. (Fricke and Powell [12]). The Taylor matrix is a $B-B$ method, that is, maps sequences of $b . i$. to sequences of b.i. for any complex number $r$.

For an entire function $f(z)$ and a sequence $\left\{z_{i}\right\}_{0}^{\infty}$ of complex numbers, define the matrix method $A\left(f, z_{i}\right)=\left(a_{n, k}\right)$ by

$$
f(z)=\sum_{k=0}^{\infty} a_{n, k}\left(z-z_{n}\right)^{k} \quad \text { for } n=0,1,2, \ldots .
$$

We can express the Silverman-Toeplitz conditions for regularity as follows (cf: $[35$, p. 23]): 
(i)

$$
\begin{array}{ll}
\text { (i) } & \lim _{n \rightarrow \infty} f^{(k)}\left(z_{n}\right)=0 \quad \text { for } k=0,1, \ldots \\
\text { (ii) } & \lim _{n \rightarrow \infty} f\left(z_{n}+1\right)=1
\end{array}
$$

and

(iii) $\sum_{k=0}^{\infty}\left|a_{n, k}\right| \leq M$ for some $M>0$ and all $n=0,1, \ldots$.

THEOREM 5.2. (Fricke and Powe11 [12]). If $f$ is of b.i.. then $A\left(f, z_{i}\right.$ ) is not regular for any sequence $\left\{z_{i}\right\}_{0}^{\infty}$.

The proof relies on the fact that if $f$ is a function of $b . i$. and $\left\{a_{n}\right\}$ is $a$ sequence of complex numbers such that $\lim _{n \rightarrow \infty} f^{(k)}\left(a_{n}\right)=0$ for all $k=0,1, \ldots$, then for any $r>0, \lim _{n \rightarrow \infty} \max _{\mid z-a_{n}} \mid=r\left\{\left|f^{(k)}(z)\right|\right\}=0$ for $k=0,1, \ldots$.

Let $A^{\prime}\left(f, z_{i}\right)=\left(b_{n, k}\right)$ denote the transpose of $A\left(f, z_{i}\right)$, that is,

$$
f(z)=\sum_{n=0}^{\infty} b_{n, k}\left(z-z_{k}\right)^{n} \quad \text { for } \quad k=0,1, \ldots .
$$

We then have the following:

THEOREM 5.3. (Fricke and Powell [12]). Let $\mathrm{f}$ be of b.i.

(i) $A^{\prime}\left(f, z_{i}\right)$ is an $\ell-\ell$ method if and only if

$$
\sup _{n}\left\{\left|f^{(k)}\left(z_{n}\right)\right|\right\}<\infty \quad \text { for } k=0,1, \ldots \text {. }
$$

(ii) $A^{\prime}\left(f, z_{i}\right)$ is an $E-E$ method if and only if for each integer $n \geq 0$ there exist an integer $p>0$ and a constant $M>0$ such that

$$
\left|f^{(n)}\left(z_{k}\right)\right| \leq p^{k} M \quad \text { for } k=0,1 \ldots \text {. }
$$

The part (ii) of this theorem does not necessarily hold for functions of exponential type and unbounded index.

THEOREM 5.4. (Fricke and Powell [12]). Let $f$ be of $b . i$. If either $A\left(f, z_{i}\right)$ or $A^{\prime}\left(f, z_{i}\right)$ is an $\ell-\ell$ method then $A^{\prime}\left(f, z_{i}\right)$ is an $E-E$ method.

In a recent paper and its corregendum ([43]) Sridhar further examines the $A\left(f, z_{i}\right)$ matrix transformation and obtains results which can be summarized as follows.

THEOREM 5.5. (Sridhar [43]). Let $\mathrm{f}$ be of b.i. Then the following are equivalent.
(i) $\quad A\left(f, z_{i}\right)$ is a $c_{0}-E$ method.
(ii) $A\left(f, z_{i}\right)$ is a $c_{0}-\chi$ method. 
(iii) $A\left(f, z_{i}\right)$ is a $c-E$ method.

(iv) $\left|f^{(k)}\left(z_{n}\right)\right|^{\frac{1}{n}}=\circ(n)$ as $n \rightarrow \infty$ for all $k=0,1, \ldots$.

THEOREM 5.6. (Sridhar [43]). Let $f$ be of $b . i$. Then $A\left(f, z_{i}\right.$ ) is a $c-E * \operatorname{method}$ if and only if

$$
\left|f^{(k)}\left(z_{n}\right)\right|^{\frac{1}{n}}=0(n) \text { as } n \rightarrow \infty \text { for all } k=0,1, \ldots \text {. }
$$

6. FUNCTIONS DEFINED BY DIRICHLET SERIES AND FUNCTIONS OF SEVERAL VARIABLES.

(i) Let

$$
f(s)=\sum_{n=0}^{\infty} a_{n} \exp \left(s \lambda_{n}\right), \lambda_{0} \geq 0, \lambda_{n+1}>\lambda_{n}
$$

be absolutely convergent everywhere and such that $\lim \inf { }_{n \rightarrow \infty}\left(\lambda_{n+1}-\lambda_{n}\right)>0$. Azpeitia [1] considers entire functions $f(s)$ and proves that if $f(s)$ is of bounded index $\mathrm{N}$, then it reduces to an exponential polynomial. Bajpai [2] replaces the condition of $b . i$. of $f(s)$ by four conditions and proves that if any one of these four conditions is satisfied then $f(s)$ reduces to an exponential polynomial. Gross [15] and Shah and Sisarcick [41] have considered similar conditions for functions defined by Taylor series.

(ii) In [32] Salmassi considers functions $f(z)=f\left(z_{1}, z_{2}\right)$ of two variables and proves the following:

THEOREM 6.1. (Salmassi [32]). Let $f(z)$ be of b.i. and a $\epsilon \phi$. Then $g(z)=$ $f(a z)$ is also of b.i.

He also obtains a necessary and sufficient condition for $f(z)$ to be of b.i. A similar theorem for a function of one variable is due to Fricke [9].

\section{REFERENCES}

1. Azpeitia, A. G. On entire functions of bounded index defined by Dirichlet expansions, Riv. Mat. Univ. Parma (4) 3, 95-97.

2. Bajpai, S. K. On entire functions of bounded index defined by Dirichlet expansions, Indian J. Pure Appl. Math. 11 (1980), 422-427.

3. Beauchamp, J. P. Inégalités Différentielles et Distribution des Valuers en Analyse Complexe, Doctoral Dissertation, University of Montreal, 1978.

4. Bohmer, K. Die möglichen Wachstumsordnungen der Lösungen von linearen Differentialgleichungen, Manuscripta Math. 4 (1971), 373-409. 
5. Frank, G. Picardsche Ausnahmewerte bei Lösungen linearer Differentialgleichungen, Dissertation, Karlsruhe, 1969.

6. Frank, G. Zur lokalen Werteverteilung der Lösungen linearer Differentialgleichungen, Manuscripta Math. 6 (1972), 381-404.

7. Frank, G. and E. Mues. Über den Index der Lösungen 1inearer Differentia1gleichungen, Manuscripta Math. 5 (1971), 155-163.

8. Frank, G. and E. Mues. Über das Wachstum des Index ganzer Funktionen, Math. Ann. 195 (1972), 114-120.

9. Fricke, G. H. A characterization of functions of bounded index, Indian J. Math. 14 (1972), 207-212.

10. Fricke, G. H. Functions of bounded index and their logarithmic derivatives, Math. Ann. 206 (1973), 215-223.

11. Fricke, G. H. and S. M. Shah. Entire functions satisfying a linear differential equation, Indag. Math. 37 (1975), 39-41.

12. Fricke, G. H. and R. E. Powe11. Bounded index and summability methods, J. Austr. Math. Soc. 21(Series A) (1976), 79-87.

13. Fricke, G. H. and S. M. Shah. On bounded value distribution and bounded index, J. of Nonlinear Analysis 2 (1978), 423-436.

14. Fricke, G. H. A note on bounded index and bounded value distribution, Indian J. Pure App1. Math. 11 (1980), 428-432.

15. Gross, F. Entire fuctions of exponential type, J. Res. Nat. Bur. Stand. (U.S.) 74B (Math. Sci.), (1970), 53-59.

16. Hayman, W. K. Research Problems in Function Theory, Athlone Press, London 1967.

17. Hayman, W. K. Differential inequalities and local valency, Pacific J. Math. 44 (1973), 117-137.

18. Heath, L.F. Vector-valued entire functions of bounded index satisfying a differential equation, J. of Research of Nat. Bur. Stand. (U.S.) 83, (1978), 75-79.

19. Hennekemper, W. Einige Ergebnisse über Ideale in Ringen ganzer Funktionen mit Wachstumsbeschränkung, Doctoral Dissertation, Fernuniversität Dortmund (1978).

20. Hennekemper, W. Some results of functions of bounded index, Lecture Notes in Math. 747, Springer-Verlag (1978), 158-160.

21. Hörmander, L. Generators for some rings of analytic functions, Bull. Amer. Math. Sc. 73, (1967), 943-949.

22. Ince, E. L. Ordinary Differential Equations, Longmans, Green and Co., London 1927.

23. Kelleher, J. J. and B. A. Taylor. Finitely generated ideals in rings of analytic functions, Math. Ann. 193, (1971), 225-237.

24. Knab, 0. Über lineare Differentialgreichungen mit rationalen Koeffizienten, Dissertation, Karlsruhe 1974. 
25. Knab, 0. Wachstumsordnung und Index der Lösungen Linearer Differentialgleichungen mit Rationalen Koeffizienten, Manuscripta Math. 18, (1976), 299-316 .

26. Knab, 0. Über das Anwachsen der Lösungen linearer Differentialgleichungen mit Rationalen Koeffizienten in Winkelraumen, Manuscripta Math. 24, (1978), 295-322.

27. Knab, 0. ther Wachstumsordnung und Typus der Lösungen linearer Differentialgleichungen mit Rationalen Koeffizienten, Archiv der Math. 31, (1978), 61-69.

28. Kövari, T. A note on entire functions, Acta. Math. Acad. Sc. Hungaricae, VIII, $(1957), 87-90$.

29. Lee, Boo-sang and S. M. Shah. An inequality involving the Bessel function and its derivatives, J. Math. Anal. and App1. 30, (1970), 144-155.

30. Lepson, B. Differential equations of infinite order, hyperdirichlet series and entire functions of bounded index, Proc. Sympos. Pure Math. Vol. XI, Amer. Math. Soc., Providence, R.I. (1968), 298-307.

31. Maríc, V. and S. M. Shah. Entire functions defined by gap power series and satisfying a differential equation, Tohoku Math. J. (2), 21, (1969), 621-631.

32. Mohammad Salmassi, Some classes of entire functions of exponential type in one and several complex variables, Doctoral Dissertation 1978, University of Kentucky.

33. Nikolaus, J. Lineare Differentialgleichungen in Komplexen, 5. Steiermarkisches Math. Symposium Stift Rein 1973 Bericht N, 1 (1973), III/1-III/16.

34. Pösch1, K. Über Anwachsen und Nullstellenverteilung der ganzen transzendenten Lösungen linearer Differentialgleichungen I, J. Reine und Angew. Math. 199, (1958), 121-138.

35. Powe11, R. E. and S. M. Shah. Summability Theory and Applications, Von Nostrand, London 1972 .

36. Shah, S. M. A note on the derivatives of intégral functions, Bul1. Amer. Math. Soc. 53, (1947), 1156-1163.

37. Shah, S. M. Entire functions of bounded index, Proc. Amer. Math. Soc. 19, (1968), 1017-1022.

38. Shah, S. M. Entire functions satisfying a linear differential equation, J. Math. Mech. 18, (1968-69), 131-136.

39. Shah, S. M. Entire functions of unbounded index and having simple zeros, Math. Zeit. 118, (1970), 193-196.

40. Shah, S. M. Entire functions of bounded index, Lecture Notes in Math., SpringerVerlag, Vo1. 599, (1977), 117-145.

41. Shah, S. M. and W. C. Sisarcick. On entire functions of exponential type, J. Res. Nat. Bur. Stand. (U.S.) 75B, No. 3, (1971), 141-147.

42. Shah, S. N. Functions of exponential type are differences of functions of bounded index, Canad. Math. Bul1. 20, (1977), 479-483.

43. Sridhar, S. Bounded Index Summability Methods, Indian J. Pure and Appl. Math. 10(2), (1979), 161-165; Corrigendum 910-911. 
44. Tang Hsiung. Index of Bessel functions and order results related to Bessel series, Doctoral Dissertation, University of Kentucky, 1977.

45. Valiron, G. Lectures on the General Theory of Integral Functions, Chelsea, New York 1949.

46. Vijayaraghavan, $T$. On derivatives of integral functions, J. London Math. Soc. 10, (1935), 116-117.

47. Wittich, H. Neuere Untersuchungen über Eindeutige Analytishe Funktionen, Springer-Verlag, Berlin 1955. 


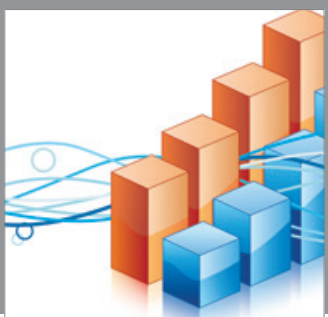

Advances in

Operations Research

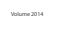

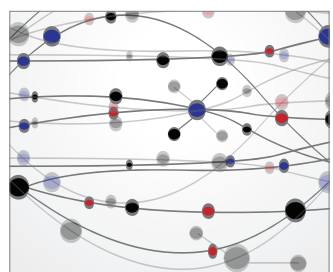

\section{The Scientific} World Journal
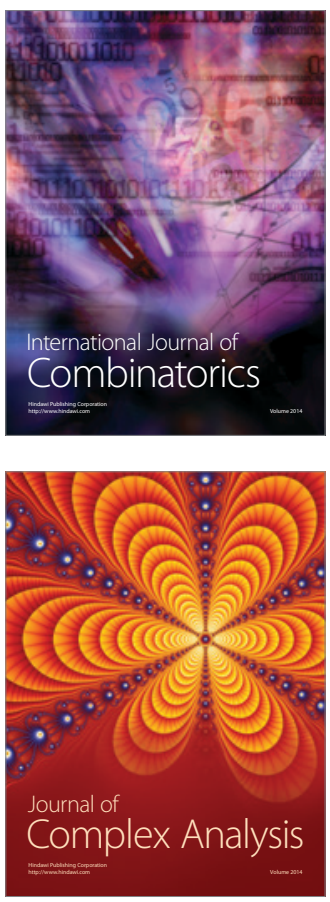

International Journal of

Mathematics and

Mathematical

Sciences
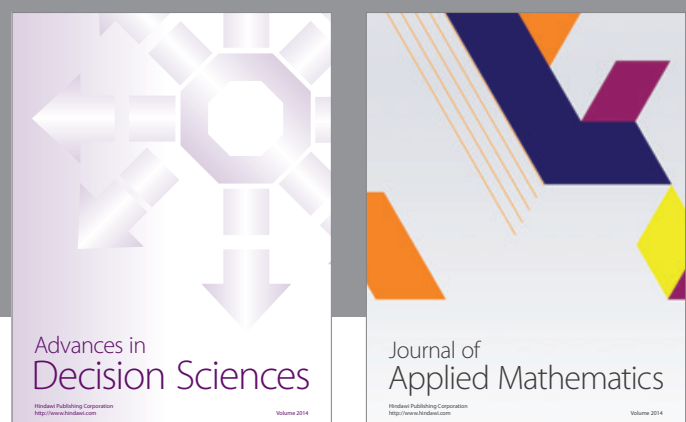

Journal of

Applied Mathematics
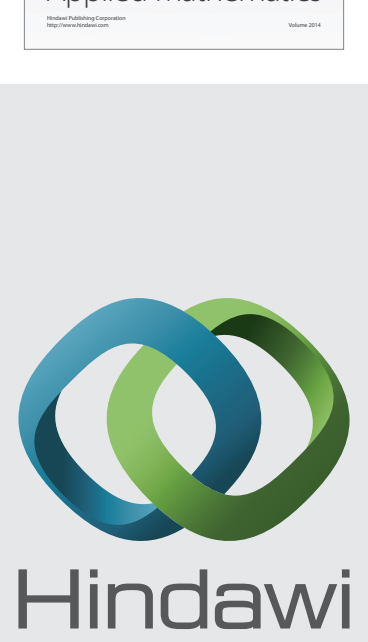

Submit your manuscripts at http://www.hindawi.com
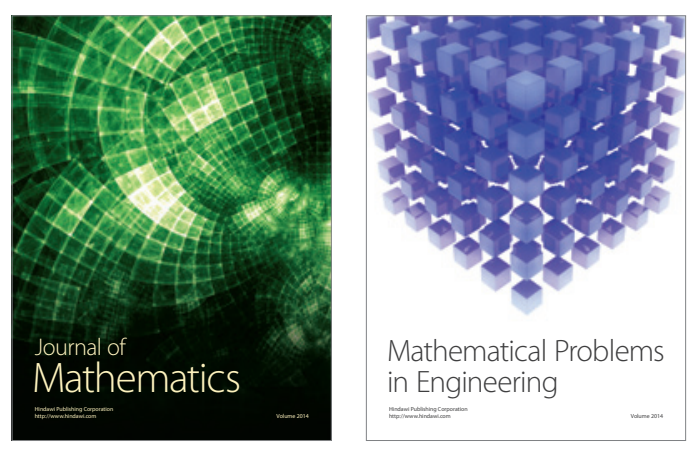

Mathematical Problems in Engineering
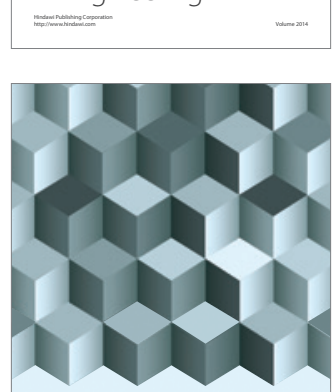

Journal of

Function Spaces
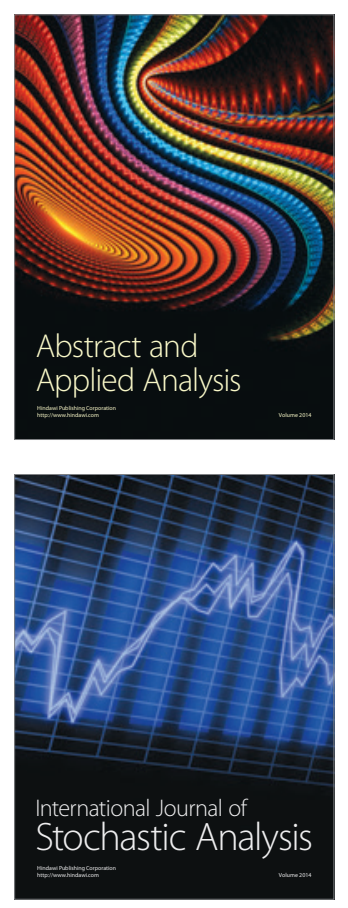

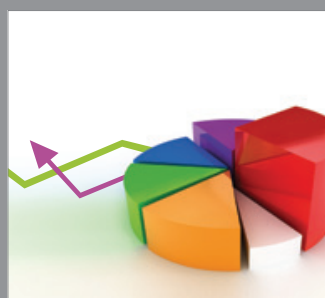

ournal of

Probability and Statistics

Promensencen
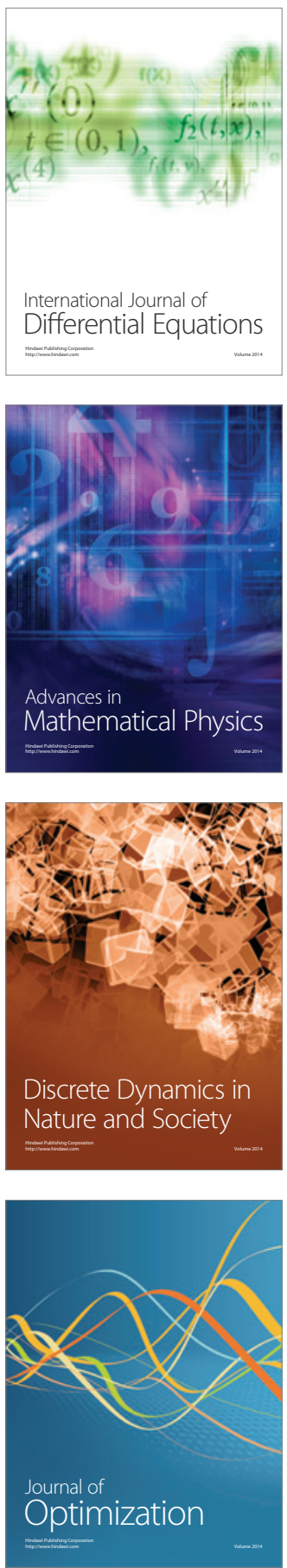